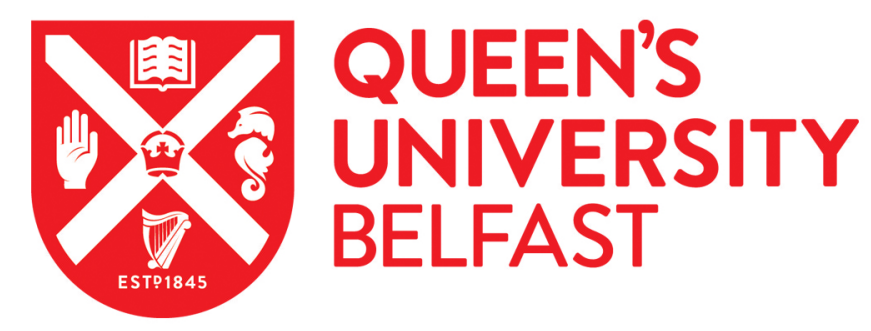

\title{
Pain catastrophizing as a treatment process variable in cognitive behavioral therapy for adults with chronic pain
}

Gilliam, W. P., Schumann, M. E., Cunningham, J. L., Evans, M. M., Luedtke, C. A., Morrison, E. J., Sperry, J. A., \& Vowles, K. E. (2021). Pain catastrophizing as a treatment process variable in cognitive behavioral therapy for adults with chronic pain. European Journal of Pain, 25(2), 339-347. https://doi.org/10.1002/ejp.1671

Published in:

European Journal of Pain

Document Version:

Peer reviewed version

Queen's University Belfast - Research Portal:

Link to publication record in Queen's University Belfast Research Portal

Publisher rights

Copyright 2020 Wiley. This work is made available online in accordance with the publisher's policies. Please refer to any applicable terms of use of the publisher.

\section{General rights}

Copyright for the publications made accessible via the Queen's University Belfast Research Portal is retained by the author(s) and / or other copyright owners and it is a condition of accessing these publications that users recognise and abide by the legal requirements associated with these rights.

Take down policy

The Research Portal is Queen's institutional repository that provides access to Queen's research output. Every effort has been made to ensure that content in the Research Portal does not infringe any person's rights, or applicable UK laws. If you discover content in the Research Portal that you believe breaches copyright or violates any law, please contact openaccess@qub.ac.uk. 
Running Head: Pain Catastrophizing

Pain catastrophizing as a treatment process in cognitive behavioral therapy for adults with chronic pain

Wesley P. Gilliam, ${ }^{\mathrm{a}}$ Matthew E. Schumann, ${ }^{\mathrm{a}}$ Julie L. Cunningham, ${ }^{\mathrm{b}}$ Michele M. Evans, ${ }^{\mathrm{a}}$ Connie A. Luedtke, ${ }^{a}$ Eleshia J. Morrison, ${ }^{a}$ Jeannie A. Sperry ${ }^{\text {a }}$ \& Kevin E. Vowles ${ }^{c}$

${ }^{a}$ Department of Psychiatry and Psychology, Mayo Clinic College of Medicine, Rochester, Minnesota, USA

${ }^{b}$ Department of Pharmacy, Mayo Clinic College of Medicine, Rochester, Minnesota, USA

c School of Psychology, Queen's University Belfast, Belfast, United Kingdom

\section{Corresponding Author:}

Wesley P. Gilliam

Mayo Clinic

Department of Psychiatry and Psychology

200 First Street SW

Rochester, MN 55905

USA

Phone: 507-255-5921; Fax: 507-284-3933

E-mail: gilliam.wesley@mayo.edu

Manuscript Category: Original Article

Authors have no conflicts of interest to report

Funding source: none declared

What is already known about this topic?

- Interdisciplinary CBT for chronic pain is effective in reducing pain catastrophizing and improving functioning and mood among individuals with chronic pain.

What does this study add?

- Patterns of change in pain catastrophizing over the course of three weeks of interdisciplinary CBT for chronic pain were related to treatment outcome at posttreatment. 
Authors Contribution: All authors discussed the results and commented on the manuscript. 


\section{Introduction}

Chronic pain is major health care problem occurring in an estimated 100 million United States citizens with an economic cost of over $\$ 600$ billion annually (Institute of Medicine, 2011). It is commonly associated with significant difficulties across emotional, occupational, social and self-care activities among sufferers. Psychosocial interventions for chronic pain have reasonable support for efficacy and represent an attractive treatment option to medical intervention (Vowles and McCracken 2008; Woods and Amundson 2008; Smeets et al., 2009; Ehde et al., 2014; Ardito et al., 2017).

Cognitive-behavioral therapy (CBT) is a widely accepted psychosocial approach for chronic pain and is commonly integrated into interdisciplinary pain rehabilitation programs. The rationale for integration of CBT is rooted in behavioral theory (Fordyce, 1976; Gatchel et al., 2014) and research indicating that an individual's appraisal of, and subsequent response to chronic pain influences physical and psychological adjustment (Burns et al., 2015). These programs are effective in promoting restoration, and reducing pain severity, medication use, and healthcare utilization (Townsend et al., 2008; Sletten et al., 2015; Gilliam et al., 2018).

A theorized active ingredient of CBT is the process of cognitive change in terms of maladaptive cognitions. Given the empirical support for the effectiveness of CBT, a critical next step is to examine how CBT works for individuals with chronic pain. If putative therapeutic processes variables can be identified and empirically supported, efforts can be directed towards more precisely targeting these variables in future interventions to presumably enhance treatment effect. This will also help translate effective treatments to real-world clinical practice by elucidating critical treatment components.

Pain catastrophizing is a robust predictor of chronic pain outcome (Severeijns et al., 2001; Sullivan et al., 2001). Longitudinal studies show that treatment-related improvements in 
pain catastrophizing are associated with improvements in pain-related functioning (Jensen et al., 2001; Thorn et al, 2007; Turner et al., 2007). Further evidence from longitudinal cross-lagged analysis suggests early changes in pain catastrophizing predict subsequent changes in pain severity and interference in multidisciplinary pain treatment programs (Burns et al., 2003a; Burns et al., 2003b). These findings indicate changes in pain catastrophizing precede and predict change in outcome, supporting an argument for pain catastrophizing as a cognitive process variable in CBT treatment. However, these cross-lagged studies are limited by relatively low sample sizes and the statistical approach does not allow for both within- and between-person assessment of change over time. Further, these analyses are not able to examine whether there are distinct patient groups based on change trajectories - for example, it seems plausible that a group of individuals may evidence decreased pain catastrophizing, while another group evidence no change.

In this non-randomized cohort study, we utilized latent growth curve modeling (LGCM) and Growth Mixture Modeling (GMM) to test the validity of pain catastrophizing as a cognitive process variable in a three-week interdisciplinary CBT of chronic pain. The aim of this study is to evaluate pain catastrophizing change trajectories during treatment and the relevance of change trajectories to outcomes at posttreatment.

\section{Methods}

\section{Participants}

Participants included individuals with chronic pain admitted to the Mayo Clinic Pain Rehabilitation Center (PRC) for a three-week course of interdisciplinary CBT for chronic pain between the dates of January 2017 and October 2018. All participants completed a preadmission assessment to determine eligibility for inclusion. Exclusion criteria included: 1) 
evidence of problematic opioid or other substance use requiring substance use treatment prior to program participation; 2) active suicidality with verbalized plan and intent for self-harm; 3) an active psychotic or mood disorder that required immediate psychiatric management at a higher level of care, or symptom severity sufficient enough to impact active participation the programming; or 4) unwillingness to participate in a group-based outpatient program of treatment. When individuals met inclusion criteria, agreed to initiate treatment, and had payment approval, they were scheduled to begin treatment.

This study was approved by the institutional review board at the Mayo Clinic in Rochester, MN and informed consent was completed by all participants prior to treatment's beginning. Participants completed a battery of self-report measures at treatment admission and discharge. A measure of pain catastrophizing, described below, was completed weekly over the three weeks of treatment. Outcome measures included pain interference, depressive symptoms, and physical and mental health quality of life (QOL). Thirty-five individuals dropped out of treatment early (7\%), thus analyses used data from the 463 individuals who completed treatment. The primary pain diagnoses of the sample were variable. Low back pain comprised the largest diagnostic category $(n=112,22.2 \%)$, with fibromyalgia $(n=95,20.5 \%)$ and headache pain $(n=$ $71,15.3 \%$ ) comprising the next two largest diagnostic groups. Descriptive information appears in Table 1.

\section{Treatment Program}

The Mayo Clinic Pain Rehabilitation Center (PRC) is a group-based, intensive, outpatient interdisciplinary pain rehabilitation program focusing on functional restoration (Fordyce, 1979; Gatchel et al., 2014). Patients admitted into the PRC have generally received extensive medical care and experienced incomplete symptom relief from multiple pharmacologic trials, surgical 
procedures and interventional pain procedures. The PRC combines functional restoration with $\mathrm{CBT}$ as its chief components. The underlying treatment philosophy emphasizes that when meaningful pain reduction is not possible, treatment approaches must shift towards maximizing functioning. PRC entails concurrent treatment by multiple disciplines including physicians, psychologists, physical therapists, nurses and clinical nurse specialists, counselors, occupational therapists, pharmacists, and dieticians, all of whom offer convergent expertise with a balance of overlapping and distinct knowledge.

The PRC treatment program is 15 days in duration with rolling admission to allow patients entry into the program as soon as is feasible. The daily program census is typically between 24 to 27 patients. Patients attend programming for 8 hours daily for 15 consecutive work days (i.e., Monday - Friday) and participate in daily physical therapy, occupational therapy, and individual and group-based CBT sessions. Each CBT session is 60 minutes in duration and follows an empirically supported treatment protocol for pain which include topics such as neuroscience of pain education, restructuring of maladaptive pain appraisals, relaxation training, activity pacing, and mood/anxiety management. See figure 1 for an example of a treatment day. Physician and pharmacist supervised opioid tapering is also executed for patients that enter treatment prescribed opioids for pain. A more detailed description of the opioid tapering process in the PRC has been detailed elsewhere (Cunningham, et al., 2016).

\section{Measures}

Demographic and pain-related information. Participants completed a battery of self-report measures on the first and last days of treatment. Demographic information captured included gender, ethnicity, age, and education level. Pain related information included primary pain location and pain duration. 
Pain Catastrophizing. Participants completed the Pain Catastrophizing Scale (PCS) at pre, mid- (approximately day 8) and posttreatment (Sullivan et al., 1995). The PCS contains 13 items across three subscales: rumination (e.g., "I worry all the time about whether the pain will end"), magnification (e.g., "I wonder whether something serious may happen"), and helplessness (e.g., "There's nothing I can do to reduce the intensity of the pain"). Psychometric evaluation of this instrument has indicated high test-retest reliability and adequate internal consistency (Osman et al., 2000). Items are rated on a Likert-type scale from $0-4(0=$ "not at all," $1=$ "to a slight degree," 2 = "to a moderate degree," 3 = "to a great degree," 4 = all the time") with a total score range of 0-52. Higher scores suggest greater tendency to catastrophize in response to pain. Total scores greater or equal to 30 represent clinically significant levels of pain catastrophizing. In the current sample, the internal consistency of the PCS was excellent at pre-, mid- and posttreatment (range Cronbach's $\alpha=.94-.95$ ).

Measures of outcome. As noted, all other measures were completed on two occasions, pretreatment and posttreatment.

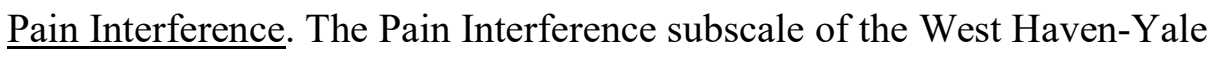
Multidimensional Pain Inventory (WHYMPI) was utilized as a primary measure of pain impact on activity (Kerns et al., 1985). The pain interference subscale is made up of 9 items, all of which are rated on a Likert-type scale from $0-6(0=$ "not at all severe," $6=$ "extremely severe"). Higher scores suggest more perceived pain-specific limitations. The pain interference subscale yielded adequate levels of internal consistency at pre- and posttreatment (Cronbach's $\alpha$ $=.91$ and .89 , respectively).

Depressive Symptoms. The Patient Health Questionnaire-9 (PHQ-9) is a 9-item measure assessing depressive symptoms (Spitzer et al., 1999). The PHQ-9 total score ranges from 0 - 27 
with severity thresholds for mild (5), moderate (10), moderately severe (15), and severe (20 or higher) depressive symptoms. Patients rate how often in the previous two weeks they experienced a variety of depressive symptoms on a Likert scale of $0-3(0=$ "not at all", $1=$ "several days", 2 = "more than half the days", 3 = "nearly every day"). The PHQ-9 has demonstrated strong test-retest reliability as well as strong discriminant validity (Kroenke et al., 2001). Responses are summed to yield a total score. The internal consistency of the current sample was appropriate at pre- and posttreatment (Cronbachs $\alpha=.82$ and.83, respectively).

Physical and Mental QOL. The Medical Outcomes Study, 36-Item Short Form Health Survey (SF-36) is a measure of 8 domains of health-related quality of life, including: general health perceptions, physical health functioning, mental health functioning, role limitations due to emotional problems, role limitations due to physical health, bodily pain, vitality, and social functioning (Ware et al., 1993). The subscales can be combined into two summary scores: physical health-related QOL and mental health QOL. Items are rated on a Likert-type scale, which are then transformed into percentages (0-100). Lower scores reflect worse QOL. Research supports strong psychometric properties for the measure, including high convergence with clinical data (McHorney et al., 1993). Internal consistency in the current sample was high at pre- and posttreatment (Physical health QOL Cronbach's $\alpha=.86$ and .91; Mental health QOL Cronbach's $\alpha=.87-.89)$, respectively.

\section{Statistical Approach}

There were three primary analytic steps. All analyses were conducted using MPlus version 8 (Muthen and Muthen, 1998-2017) and missing data was addressed using maximum likelihood (ML), which uses all available data.

First, the effectiveness of treatment was examined through posttreatment using multilevel 
structural equation modeling (SEM: Preacher et al., 2010) for the outcomes of pain interference, depression, and physical and mental health QOL. Because treatment cohort membership was not static (due to the process of rolling admission to the PRC), we were not able to examine the role of treatment cohort on outcome. Within participant effect size (Hedges' $g$ ) was also calculated.

Second, Latent Growth Curve Modeling (LGCM) analyses were conducted to determine trajectory of change and identify any latent classes of change in the participant data (i.e., participant groups) in the PCS at pre-, mid-, and posttreatment. We examined the fit of both linear and linear + quadratic trajectories of change. Model fit was examined using several indicators, including the Root Mean Square Error of Approximation (RMSEA), the Standardized Root Mean Square Residual (SRMR), and the Comparative Fit Index (CFI). Adequate model fit was defined as RMSEA of $\leq .08, \mathrm{SRMR} \leq .08$, and CFI $\geq .95$ (Bryne, 2001; Hu and Bender 1999; MacCallum et al., 1996).

Latent classes were examined by adding a categorical variable specifying the number of patient groups to be extracted from the LGCM. Additional latent classes were added until comparative analyses between models indicated a lack of improvement in fit or a failure of model convergence. Specifically, the initial analyses examined one latent class of change, followed by an examination of two latent classes of change with additional classes being examined sequentially until this addition did not provide improved model fit. Model fit was evaluated using the Bayesian information criterion (BIC: Schwarz, 1978; Nylund et al., 2007), sample size adjusted BIC (aBIC: Sclove, 1987), Vuong-Lo-Mendell-Rubin test (VLMR: Vuong, 1989; Lo et al., 2001; Asparouhov and Muthen, 2012), and entropy. For BIC and aBIC, smaller values indicate better fit. The VLMR offers a significance test for the improvement in model fit with the addition of one class (e.g., fit of a two class model in comparison to a one class model). 
Entropy ranges from $0-1$ with higher values indicating better classification precision. The best fitting latent growth trajectory and number of latent classes of change was retained for subsequent analyses.

Finally, Growth Mixture Modeling (GMM) was performed to examine whether different classes of change evidenced differential rates of improvement at post treatment on the outcome variables of pain interference, depression, and physical and mental health QOL. When significant, these analyses indicate that trajectory of change in the PCS during treatment is differentially related to amount of change in treatment outcomes. Between class comparisons used the $\mathrm{BCH}$ method, which allows a between-class significance test in relation to continuous distal outcomes (Asparouhov \& Muthén, 2014).

\section{Results}

Descriptive Details and Data Integrity. Descriptive information for study variables is presented in Table 2. With regard to sample size, 498 individuals began treatment and 463 (93.0\%) completed treatment. Attrition analyses did not indicate any significant differences between those who did and did not complete treatment for gender, age, or pain duration, nor were there differences in pre-treatment scores for any measure, including pain catastrophizing, pain interference, depression, physical health QOL, and mental health QOL.

Treatment Effectiveness. As noted, multilevel SEM was used to evaluate for change in the outcome measures across time, including pretreatment and posttreatment. Data were nested hierarchically at two levels: (1) repeated assessment over time and (2) within participants. These analyses indicated a significant effect of time on outcome at each assessment point. The effect of time was associated with decreases in pain interference and depression, $\mathrm{B}(\mathrm{SE})=-0.99(0.05), p$ $<0.001$ and $\mathrm{B}(\mathrm{SE})=-4.17(1.30), p<0.001$, respectively. It was associated with increases in 
physical and mental health functioning, $\mathrm{B}(\mathrm{SE})=11.8(0.78), p<0.001$ and $\mathrm{B}(\mathrm{SE})=5.16$ (0.81), $p<0.001$, respectively. Table 2 also displays within participants effect size, Hedges' $g$, which were large for each of the four outcome measures.

Classes of Latent Change. The LGCM analyses of a single class of change indicated poor fit with the data for the linear model $[\mathrm{RMSEA}=.24(90 \% \mathrm{CI}: .17-.31), \mathrm{SRMR}=.05, \mathrm{CFI}$ $=.93]$, while the single class linear+quadratic model was unidentified. Therefore, analyses proceeded with an examination of multiple classes of change.

As shown in Table 3, analyses indicated that a two class linear model fit significantly better than the single class linear model. Entropy was acceptable as well, indicating good separation between the two latent classes. The 2 class linear + quadratic model did not fit significantly better than a single class model. Furthermore, all models with additional latent classes failed to converge. Therefore, the 2 class linear change model was retained for the remaining analyses.

See Figure 1 for details regarding the two latent classes of change on the PCS. The first class, comprising $11.7 \%$ of the sample $(n=58)$, was characterized by a non-significant slope in PCS across the three measurement points (slope: -0.18 ; SE: $0.28, p=.51$ ). The second class, comprising $88.3 \%$ of the sample $(n=439)$, was characterized by a significant negative slope in PCS (slope: -1.96 , SE: $0.25 ; p<.001)$. These classes were labeled as the "unchanged" and "improved" groups, for class 1 and 2, respectively. As shown in Table 4, the two classes did not significantly differ with regard to gender, age, education, or pain duration. GMM Analyses. As the final analytic step, the two classes of change on the PCS were examined in relation to treatment outcomes at post-treatment using several GMMs. These analyses sought to determine if the trajectories differed from one another in terms of raw change in treatment outcome 
measures of pain interference, depression, and physical and mental health functioning. As shown in Table 4, the improved PCS group of patients had significantly greater improvements in comparison to the unchanged PCS group across all four measures of outcome at post-treatment, including pain interference, depression, and physical and mental functioning.

\section{Discussion}

The present analysis examined patterns of change in pain catastrophizing over the course of a three-week interdisciplinary CBT for chronic pain program to evaluate if aspects of change were related to posttreatment outcome. Overall, findings indicated that change in pain catastrophizing, as assessed by the PCS, was best characterized by a two class linear trajectory model. The first class was characterized by a non-significant slope in PCS (i.e., "unchanged") over the course of treatment while the second class was characterized by a significant negative slope in PCS (i.e., "improved"). While results revealed that each class of participants improved in pain-related function, the "improved" PCS class was associated with a significantly greater magnitude of improvement in pain interference, mood and quality of life when compared to the "unchanged" class. These outcomessuggest that reducing catastrophic responses to pain during interdisciplinary CBT may enhance the magnitude of effect on psychosocial outcome at the end of treatment. These findings provide support for the CBT model and the importance it places on altering cognitive content and cognitive processes, such as reducing catastrophic pain appraisals.

Examining treatment process within interdisciplinary CBT for pain is important. The CBT model specifies that altering maladaptive perceptions of and poor coping with chronic pain should promote improved functioning and mood. Therefore, the debilitating effects of pain should diminish as people with pain learn to conceptualize pain less as an unmitigated catastrophe, and more as a manageable condition. A tendency in evaluating outcomes in 
interdisciplinary CBT is to assume that when salutary pre- to posttreatment improvements in outcome are observed, that change in cognitive appraisal is the active agent that accounted for those improvements. This has been referred to this as the "Specific Mechanism Model" whereby change in outcome is believed to be due strictly to the specific therapeutic operations used in a CBT intervention (Burns et al, 2015). While findings from numerous studies exploring the effectiveness of interdisciplinary CBT treatment report pre- to posttreatment improvement in pain catastrophizing, and important pain- related outcomes such as pain adjustment, mood and quality of life, this work is predominantly limited to cross-sectional data or longitudinal data where both the process and outcome variables are collected at the same time point, preventing examination of temporal relationships (Craner et al., 2016; van der Mass et al., 2016). Furthermore, studies utilizing methodology allowing for examination of timing of change between process and outcome (e.g., pain catastrophizing) do not allow for the comparison of distinct patient groups based on process change trajectories (Burns et al., 2003a; Burns et al, 2003b).

To our knowledge, the present study is the first to have utilized LGCM and GMM to examine treatment process in an interdisciplinary CBT for chronic pain program. This statistical approach offers some advantage over cross-lagged analysis in that it not only allows for examination of the extent to which change in catastrophizing during treatment contributes to posttreatment improvement in pain outcome, but it also allows for more fine-grained analysis of change at the level of the individual patients. That is, GMM allows for examination of whether there are different groups of participants that could be classified by PCS change trajectory, and then compare these groups on outcome to determine if one trajectory is more responsive to treatment than another. 
There are several limitations that should be considered when interpreting these results. First, because this trial was uncontrolled, the effectiveness of treatment in comparison to an alternative treatment approached was not assessed. Second, the treatment model was intensive and interdisciplinary, delivered in a tertiary care setting. It is possible that the treatment findings are not generalizable to patients with chronic pain treated in a less intensive environment. Third, approximately $7 \%$ of participants dropped out before providing post-treatment data. It is possible that a more complete set of data would have resulted in different outcomes. However, the large sample size of treatment completers provides some confidence in the reliability of the findings. Of course, these findings will need to be replicated in similarly structured programs and in alternative treatment contexts to enhance confidence in both reliability and generalizability of these findings. Fourth, changes that occurred in treatment may have been better accounted for not by CBT but by other modalities included in the interdisciplinary treatment package such as physical or occupational therapy. Fifth, follow-up data was not reported, thus it was not possible to examine the role of the latent trajectories of change in PCS over the three weeks of treatment on longer term outcomes. In addition, it is possible that more frequent assessment of the PCS during treatment would have allowed for a more detailed analysis of change trajectories. Future research utilizing follow-up data is needed.

In conclusion, our findings provide evidence that reductions in the tendency to pain catastrophize over the course of interdisciplinary CBT for chronic pain constitutes a potential cognitive process variable that contributes to improvement in pain outcome. The present data indicated that slope of change in PCS was statistically useful in predicting core outcome domains of chronic pain management, which provides support for the CBT model. 


\section{References}

Ardito, R.B., Pirro, P.S., Bonapace, I., Menardo, V., Bruno, E., \& Gianotti, L. (2017) Mindfulness-based stress reduction program on chronic low-back pain: A study investigating the impact on endocrine, physical, and psychologic functioning. The Journal of Alternative and Complementary Medicine, 23, 15-623.

Asparouhov, T. \& Muthen, B.O. (2012). MPlus web notes: No14-using MPlus TECH14 to test the number of latent classes. Retrieved from http://www.starmodel.com/examples/webnotes/webnote14.pdf on November 12, 2019.

Asparouhov T, Muthén BO: MPlus Web Notes No 21 - Auxiliary variables in mixture modeling : Using the $\mathrm{BCH}$ Method in Mplus to estimate a distal outcome model and an arbitrary secondary model. 2014. Retrieved from: https://www.statmodel.com/download/asparouhov_muthen_2014.pdf on August 18, 2020. Bryne, B.M. (2001). Structural equation modeling with AMOS: Basic concepts, applications, and programming. Mahwah, NJ: Lawrence Erlbaum.

Burns, J.W., Glenn, B., Bruehl, S., Harden, R.N., \& Lofland, K. (2003). Cognitive factors influence outcome following multidisciplinary chronic pain treatment: a replication and extension of a cross-lagged panel analysis. Behaviour Research and Therapy, 41, 11631182.

Burns, J.W., Kubilus, A., Bruehl, S., \& Harden RN. (2003). Do changes in cognitive factors influence outcome following multidisciplinary treatment for chronic pain? A cross-lagged panel analysis. Journal of Consulting and Clinical Psychology, 71, 81-91. 
Burns, J.W., Nielson, W.R., Jensen, M.P., Heapy, A., Czlapinski, R., \& Kerns, R.D. (2015). Does change occur for the reasons we think it does? A test of specific therapeutic operations during cognitive-behavioral treatment of chronic pain. Clinical Journal of Pain, 31, 603-611.

Craner, J.R., Sperry, J.A., \& Evans, M.M. (2016). The relationship between pain catastrophizing and outcomes of a 3-week comprehensive pain rehabilitation program. Pain Medicine, 17, 2026-2035.

Cunningham, J.L., Evans, M.M., King, S.M., Gehin, J.M \& Loukianova, L.L. (2016). opioid tapering in fibromyalgia patients: Experience from an interdisciplinary pain rehabilitation program. Pain Medicine, 17, 1676-1685.

Department of Health and Human Services. (2011). National pain strategy: A comprehensive population health strategy for pain. Retrieved from https://iprcc.nih.gov/docs/HHSNational_Pain_Strategy.pdf. Accessed May 25, 2016.

Ehde, D.M., Dillworth, T.M. \& Turner, J.A. (2014). Cogntive-behavioral therapy for Individuals with chronic pain: Efficacy, innovations, and directions for research. American Psychologist, 69, 153-166.

Fordyce, W.E. (1976). Behavioral methods for chronic pain and illness. St. Louis, Missouri: C.V. Mosby.

Gatchel, R.J., McGeary, D., McGeary, C.A., \& Lippe, B. (2014). Interdisciplinary chronic pain management. American Psychologist, 69, 119-130. 
Gilliam, W.P., Craner, J.R., Cunningham, J.L., Evans, M.M., Luedtke, C.A., Morrison, E.J., ...Loukianova, L.L. (2018). Longitudinal treatment outcomes for an interdisciplinary pain rehabilitation program: comparisons of subjective and objective outcomes on the basis of opioid use status. Journal of Pain, 19, 678-689.

Hu, L.T. \& Bender, P.M. (1999). Cutoff criteria for fit indexes in covariance structure analysis: Conventional criteria versus new alternatives. Structural Equation Modeling, 6, $1-55$.

Jensen, M.P., Turner, J.A., \& Romano, J.M. (2001). Changes in beliefs, catastrophizing, and coping are associated with improvement in multidisciplinary pain treatment. Journal of Consulting and Clinical Psychology, 69, 655-662.

Kerns, R.D., Turk, D.C., \& Rudy, T.E. (1985). The west haven-yale multidimensional pain inventory (WHYMPI). Pain, 23, 345-356.

Kerns, R.D., Burns, J.W., Shulman, M., Jensen, M.P., Nielson, W.R., Czlapinski, R., ... Rosenberger, P. (2014). Can we improve cognitive-behavioral therapy for chronic back pain treatment engagement and adherence? A controlled trial of tailored versus standard therapy. Health Psychology, 33, 938-947.

Kroenke, K., Spitzer, R.L., \& Williams, J.B. (2001). The PHQ-9: validity of a brief depression severity measure. Journal of General Internal Medicine, 16, 606-613.

Lamb, S.E., Hansen, Z., Lall, R., Castelnuovo, E., Withers, E.J., Michols, V., ... Underwood, M.R. (2010). Group cognitive -behavioural treatment for low-back pain in 
primary care: A randomized controlled trial and cost-effectiveness analysis. Lancet, 375, 916-923.

Lo, Y., Mendell, N., \& Rubin, D. (2001). Testing the number of components in a normal mixture. Biometrika, 88, 767-778.

MacCallum, R.C., Browne, M.W., \& Sugawara, H.M. (1996). Power analysis and determination of sample size for covariance structure modeling. Psychological Methods, $1,130-149$.

McHorney, C.A., Ware, J.E., Raczek, A.E. (1993). The MOS 36-item short form health survey (SF-36): II. Psychometric and clinical test of validity in measuring physical and mental health constructs. Medical Care, 31, 247-263.

Muthen, L.K. \& Muthen, B.O. (1998-2017). Mplus User's Guide. Eighth Edition. Los Angeles, CA: Muthen \& Muthen.

Nylund, K.L., Asparouhov, T., \& Muthen, B.O. (2007). Deciding on the number of classes in latent class analysis and growth mixture modeling: A monte carlo simulation study. Structural Equation Modeling, 14, 535-569.

Osman, A., Francisco, F.X., Gutierrez, B.A.K., Merrifield, T., \& Grittmann, L. (2000). The pain catastrophizing scale: Further psychometric evaluation with adult samples. Journal of Behavioral Medicine, 23, 351-365.

Preacher, K.J., Zyphur, M.J., \& Zhang, Z. (2010). A general multilevel SEM framework for assessing multilevel mediation. Psychological Methods, 15, 209-233. 
Schwarz G (1978). Estimating the dimension of a model. The Annals of Statistics, 6, 461464.

Sclove SL. (1987). Application of model-selection criteria to some problems in multivariate analysis. Psychometrika, 52, 333-343.

Severeijns, R.M., Vlaeyen, J.W.S., van den Hout, M.A., \& Weber, W.E.J. (2001). Pain catastrophizing predicts pain intensity, disability and psychological distress independent of level of physical impairment. Clinical Journal of Pain, 17, 165-172.

Sletten, C.D., Kurklinsky, S., Chinburapa, V., \& Ghazi, S. (2015). Economic analysis of a comprehensive pain rehabilitation program: A collaboration between florida blue and mayo clinic florida. Pain Medicine, 16, 898-904.

Smeets, R.J., Severens, J.L., Beelen, S., Vlaeyen, J.W., \& Knottnerus, A.J. (2009). More is not always better: Cost effectiveness analysis of combined, single behavioral and single physical rehabilitation programs for chronic low back pain. European Journal of Pain, 13, 71-81.

Spitzer, R.L., Kroenke, K., \& Williams, J.B. (1999). Validation and utility of a self-report version of PRIME-MD. Journal of the American Medical Association, 282, 1737-1744.

Sullivan, M.J.L, Bishop, S.R., \& Pivik, J. (1995). The pain catastrophizing scale:

Development and validation. Psychological Assessment, 7, 524-532.

Sullivan, M.J.L., Thorn, B., Haythornthwaite, J.A., Keefe, F., Martin, M., ... Lefebvre, J.C. (2001). Theoretical perspectives on the relation between catastrophizing and pain. Clinical Journal of Pain, 58, 52-64. 
Thorn, B.E., Pence, L.B., Ward, L.C., Kilgo, G., Clements, K.L., Cross, T.H., ... Tsui, P.W. (2007). A randomized clinical trial of targeted cognitive behavioral treatment to reduce catastrophizing in chronic headache sufferers. Journal of Pain, 8, 938-949.

Townsend, C.O., Kerkvliet, J.L., Bruce, B.K., Rome, J.D., Hooten, W.M., ... Hodgson, J.E. (2008). A longitudinal study of the efficacy of a comprehensive pain rehabilitation program with opioid withdrawal: Comparison of treatment outcomes based on opioid use status at admission. Pain, 140, 177-189.

Turner, J.A., Holtzman, S., \& Mancl, L. (2007). Mediators, moderators , and predictors of therapeutic change in cognitive-behavioral therapy for chronic pain. Pain, 127, 276286.

Van der Mass, L.C., Koke, A., Bosscher, R.J., Twisk, J.W., Janssen, T.W, \& Peters, M. (2016). Body awareness as an important target in multidisciplinary chronic pain treatment: mediation and subgroup analyses. Clinical Journal of Pain, 32, 763-772.

Vuong, Q. (1989). Likelihood ration tests for model selection and non-nested hypotheses. Econometrica, 57, 307-333.

Vowles, K.E. \& McCracken, L.M. (2008). Acceptance and values-based action in chronic pain: A study of treatment effectiveness and process. Journal of Consulting and Clinical Psychology, 76, 397-407.

Ware, J.E., Sherbourne. C.D., \& Davies, A.R. (1993). Developing and testing the MOS 20-item short-form health survey: a general application. In A.L. Stewart \& J.E. Ware 
(Eds.), Measuring Functioning and Well-Being: The Medical Outcomes Study Approach (pp270-290). North Carolina, Duke University Press.

Woods, M.P. \& Asmundson, G.J. (2008). Evaluating the efficacy of graded in vivo exposure for the treatment of fear in patients with chronic back pain: A randomized controlled clinical trial. Pain, 136, 271-280. 
Table 1.

Daily Pain Rehabilitation Center Schedule

\begin{tabular}{ll}
\hline Time & Activity \\
\hline 8:00am-9:00am & Morning stretch and daily goal setting \\
\hline 9:00am-10:00am & CBT for pain group \\
\hline $10: 00 \mathrm{am}-11: 00 \mathrm{am}$ & Tai Chi or Yoga group plus relaxation \\
\hline $11: 00 \mathrm{am}-12: 00 \mathrm{pm}$ & OT group \\
\hline $12: 00 \mathrm{pm}-1: 00 \mathrm{pm}$ & Lunch hour \\
\hline $1: 00 \mathrm{pm}-2: 00 \mathrm{pm}$ & CBT for Pain group \\
\hline 2:00pm-3:00pm & Medication education group \\
\hline $3: 00 \mathrm{pm}-4: 00 \mathrm{pm}$ & PT Group \\
\hline
\end{tabular}


Table 1

Participant Characteristics $(\mathrm{N}=463)$

Age (years)

Gender (female)

Education

Race/ethnicity

Marital Status

Duration of Pain (years)

Primary Pain Location

$20.5 \%$ Fibromyalgia

$15.3 \%$ Headache

$14.0 \%$ Pain in three or more locations

$5.6 \%$ Abdominal

$20.4 \%$ Other 
Table 2

Means (SDs) and Effect Size (ES; Hedges' g) for outcome variables

\begin{tabular}{lccc}
\hline Measure & Pre-Treatment & Post-Treatment & ES \\
\hline Pain Interference & $4.5(1.1)$ & $3.0(1.4)$ & 1.20 \\
Depression & $12.6(6.0)$ & $5.6(4.5)$ & 1.25 \\
Physical Health QOL & $35.3(17.0)$ & $65.9(18.9)$ & 1.60 \\
Mental Health QOL & $30.3(15.2)$ & $67.1(20.9)$ & 1.71
\end{tabular}

Note: Pain Interference assessed with the West Haven Yale Multidimensional Pain Inventory

Pain Interference subscale; Depression assessed with Patient Health Questionnaire-9; Physical and Mental Health Quality of Life assessed with the SF-36. 
Table 3

Criteria used to assess fit for number of classes and trajectory of change in the Pain Catastrophizing Scale.

\begin{tabular}{|c|c|c|c|c|c|c|c|c|}
\hline \multirow{3}{*}{$\begin{array}{c}\# \\
\text { classe }\end{array}$} & \multicolumn{4}{|c|}{$\underline{\text { Linear }}$} & \multicolumn{4}{|c|}{ Quadratic } \\
\hline & $\mathrm{BIC}$ & $\mathrm{aBIC}$ & VLMR & Entropy & $\mathrm{BIC}$ & $\mathrm{aBIC}$ & VLMR & Entropy \\
\hline & & & & & & & & \\
\hline 1 & 10564 & 10539 & $\mathrm{n} / \mathrm{a}$ & $\mathrm{n} / \mathrm{a}$ & \multicolumn{4}{|c|}{ Model unidentified } \\
\hline \multirow[t]{3}{*}{2} & 10471 & 10436 & 2v1: & .87 & 10496 & 10564 & $2 \mathrm{v} 1: 20.7$ & .58 \\
\hline & \multicolumn{4}{|c|}{$106.4, p<$} & & \multicolumn{3}{|c|}{$p=.24$} \\
\hline & \multicolumn{4}{|c|}{.001} & & & & \\
\hline
\end{tabular}

NOTE $:$ BIC $=$ Bayesian Information Criteria, aBIC $=$ Sample-size adjusted BIC $;$ VLMR = Vuong-Lo-Mendell-Rubin Likelihood Ratio Test for $k$ versus $k$-1 classes. 
Table 4

Pain catastrophizing trajectory class comparisons for baseline characteristics and change in posttreatment outcomes. Means (SE's) in units of raw change are displayed.

Measure

\begin{tabular}{|l|c|c|c|}
\hline$\underline{\text { Class 1 }}$ & $\underline{\text { Class 2 }}$ & \\
(unchanged PCS) & $\underline{\text { (improved PCS) }}$ & $\underline{p}$ \\
\hline
\end{tabular}

\begin{tabular}{l|c|c|c|}
\hline Gender ( \% female) & $63 \%$ & .32 \\
\hline Age & $50.9(2.3)$ & $47.6(0.7)$ & .19 \\
\hline Education (yrs.) & $14.7(0.6)$ & $15.5(.1)$ & .19 \\
\hline Pain Duration (yrs.) & $8.3(1.4)$ & $10.8(0.5)$ & .11 \\
\hline
\end{tabular}

Change in Post-Treatment Outcomes

\begin{tabular}{|l|c|c|c|}
\hline Pain Interference & $-0.5(0.1)$ & $-1.7(0.1)$ & $<.001$ \\
\hline Depression & $-4.6(0.9)$ & $-7.4(0.3)$ & $<.01$ \\
\hline Physical Health & $15.5(2.7)$ & $32.4(1.0)$ & $<.001$ \\
\hline Mental Health & $20.3(3.6)$ & $39.0(1.1)$ & $<.001$ \\
\hline
\end{tabular}

Note: Pain Interference assessed with the West Haven Yale Multidimensional Pain Inventory

Pain Interference subscale; Depression assessed with Patient Health Questionnaire-9; Physical Health and Mental Health assessed with the SF-36. 
